\title{
Cardiac contraction activates endocardial Notch signaling to modulate chamber maturation in zebrafish
}

\author{
Leigh Ann Samsa ${ }^{1,2}$, Chris Givens ${ }^{1,2}$, Eleni Tzima ${ }^{1,2,3}$, Didier Y. R. Stainier ${ }^{4,5}$, Li Qian²,6 and Jiandong Liu²,4,6,*
}

\begin{abstract}
Congenital heart disease often features structural abnormalities that emerge during development. Accumulating evidence indicates a crucial role for cardiac contraction and the resulting fluid forces in shaping the heart, yet the molecular basis of this function is largely unknown. Using the zebrafish as a model of early heart development, we investigated the role of cardiac contraction in chamber maturation, focusing on the formation of muscular protrusions called trabeculae. By genetic and pharmacological ablation of cardiac contraction, we showed that cardiac contraction is required for trabeculation through its role in regulating notch $1 \mathrm{~b}$ transcription in the ventricular endocardium. We also showed that Notch1 activation induces expression of ephrin b2a (efnb2a) and neuregulin 1 ( $n r g 1$ ) in the endocardium to promote trabeculation and that forced Notch activation in the absence of cardiac contraction rescues efnb2a and $n r g 1$ expression. Using in vitro and in vivo systems, we showed that primary cilia are important mediators of fluid flow to stimulate Notch expression. Together, our findings describe an essential role for cardiac contraction-responsive transcriptional changes in endocardial cells to regulate cardiac chamber maturation.
\end{abstract}

KEY WORDS: Cardiac chamber maturation, Trabeculation, Blood flow, Cardiac contraction, Notch, Neuregulin 1, Zebrafish, Primary cilia

\section{INTRODUCTION}

Congenital heart diseases often feature structural abnormalities that arise from defects in the development of the heart during embryogenesis (Chin et al., 2012; Samsa et al., 2013). The heart is the first organ to function, yet the details of its formation are only partly understood. In vertebrates, cardiac morphogenesis commences as the two bilateral cardiac primordia fuse at the ventral midline to form the linear heart tube, which is composed of a luminal endocardial layer and an immature myocardial layer (Fishman and Chien, 1997; Harvey, 2002; Olson and Srivastava, 1996; Staudt and Stainier, 2012; Yelon, 2001). Concomitant with cardiac contraction, the primitive heart tube develops into a multichambered functional organ that grows and matures through a series of complex morphogenic processes collectively known as

\footnotetext{
${ }^{1}$ Department of Cell Biology and Physiology, University of North Carolina at Chape Hill, Chapel Hill, NC 27599, USA. ${ }^{2}$ McAllister Heart Institute, UNC School of

Medicine, University of North Carolina at Chapel Hill, Chapel Hill, NC 27599, USA. ${ }^{3}$ Division of Cardiovascular Medicine, Radcliffe Department of Medicine, University of Oxford, Oxford OX3 7BN, UK. ${ }^{4}$ Department of Biochemistry and Biophysics, University of California, San Francisco, CA 94158, USA. ${ }^{5}$ Department of Developmental Genetics, Max Planck Institute for Heart and Lung Research, Bad Nauheim 61231, Germany. ${ }^{6}$ Department of Pathology and Laboratory Medicine, University of North Carolina at Chapel Hill, Chapel Hill, NC 27599, USA.

*Author for correspondence (jiandong_liu@med.unc.edu)
}

Received 27 April 2015; Accepted 15 October 2015 cardiac chamber maturation (Moorman and Christoffels, 2003; Samsa et al., 2013).

As a part of chamber maturation, cardiac trabeculation is a tightly regulated process by which ventricular cardiomyocytes protrude and expand into the lumen of the ventricular chambers to form ridge-like muscular structures called cardiac trabeculae (Liu et al., 2010; Peshkovsky et al., 2011). Trabeculae increase cardiac output and allow for nutrition and oxygenation of the myocardium before coronary vascularization and are required for establishment of the mature conduction system of the developing ventricle (Lai et al., 2010; Liu et al., 2010). Thus, failure to form cardiac trabeculae causes embryonic lethality, and subtle perturbations of this process could lead to congenital cardiomyopathy (Jenni et al., 1999).

Crosstalk between endocardial and myocardial cells is important for cardiac maturation. Zebrafish cloche mutants that do not form endocardial cells fail to develop trabeculae and ultimately die, presumably from heart failure (Peshkovsky et al., 2011; Stainier et al., 1995). Mice deficient in the epidermal growth factor (EGF) receptor ligand neuregulin 1 ( $\mathrm{Nrg} 1)$, which is expressed in the endocardium and signals through the myocardial ErbB4-ErbB2 receptor complex, fail to form trabeculae (Gassmann et al., 1995; Lee et al., 1995; Meyer and Birchmeier, 1995). Likewise, inhibition of Nrg1-ErbB signaling in zebrafish embryos completely blocks trabeculation (Liu et al., 2010; Peshkovsky et al., 2011; Samsa et al., 2013; Staudt et al., 2014). Notch ligands and receptors are expressed in endocardial cells during development, and Notch signaling regulates many aspects of endothelial biology, including artery-vein specification, angiogenesis and proliferation (Benedito and Hellstrom, 2013; Corada et al., 2014; de la Pompa and Epstein, 2012; Gridley, 2010). Upon ligand binding, the cleaved Notch receptor intracellular domain (NICD) translocates to the nucleus, where it acts as a co-factor to promote transcription of Notch effectors, including ephrin B2, an essential upstream regulator of Nrg1 signaling (Grego-Bessa et al., 2007). Despite this knowledge, questions remain on whether this epistasis is a requirement for all vertebrate cardiac trabeculation, the precise spatiotemporal roles of these genes and the roles of mediators upstream of Notch.

An increasing body of evidence suggests that the biomechanical forces generated by the functioning embryonic heart could influence cardiac chamber maturation, underscoring the importance of a dynamic relationship between cardiac form and cardiac function (Auman et al., 2007; Bartman et al., 2004; Dietrich et al., 2014; Hove et al., 2003; Kalogirou et al., 2014; Lin et al., 2012; Peralta et al., 2013; Stainier et al., 2002; Vermot et al., 2009; Yang et al., 2014). Interestingly, in zebrafish and chick embryos, reducing blood flow through the ventricle significantly impairs cardiac trabeculation (Peshkovsky et al., 2011; Sedmera et al., 1999; Staudt et al., 2014). Nonetheless, how the mechanical stimulus is sensed and translated into spatial and temporal signals to regulate cardiac trabeculation through regulatory interaction with other myocardial signals remains largely unexplored. 

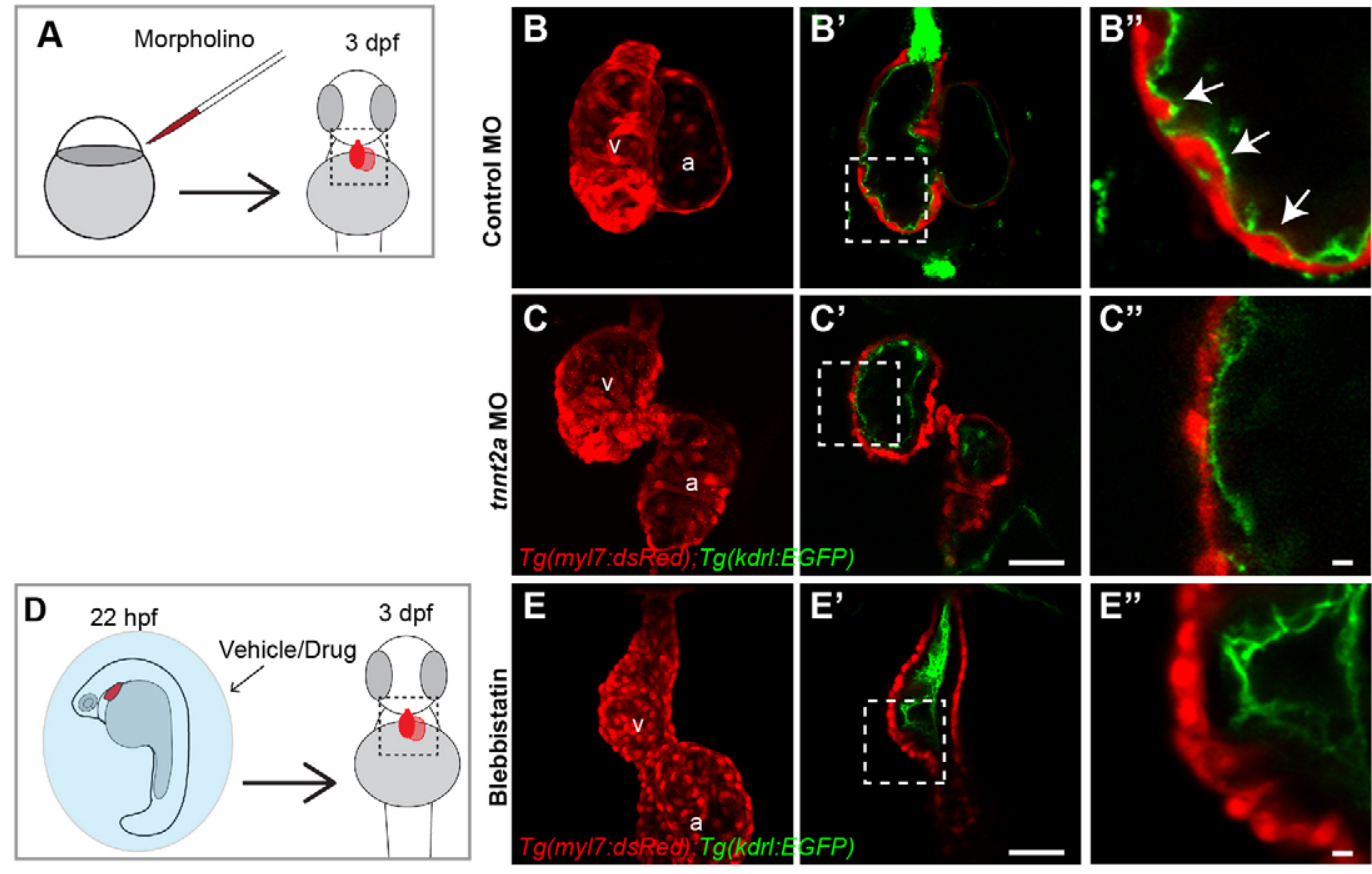

Fig. 1. Cardiac contraction is required for myocardial trabeculation. (A,D) Schematic diagrams of $(A)$ morpholino (MO) injection at one-cell stage or (D) pharmacological inhibition of cardiac contraction (6 $\mathrm{MM}$ blebbistatin) from $22 \mathrm{hpf}$. The morpholino-injected or chemical-treated $T g(k d r l: E G F P) ; T g(m y / 7$ : $d s R e d$ ) double transgenic embryos were allowed to develop further and examined for cardiac trabecular phenotypes at $3 \mathrm{dpf}$. (B,C,E) Maximal projection of confocal z-stacks reveals the overall shape of the heart. $\left(B^{\prime}, C^{\prime}, E^{\prime}\right)$ Mid-chamber confocal optical section of the same hearts shown in $B, C, E$. $\left(B^{\prime \prime}, C^{\prime \prime}, E^{\prime \prime}\right)$ Magnified high-resolution images of the cardiac regions marked by dotted lines in $\mathrm{B}^{\prime}, \mathrm{C}^{\prime}, \mathrm{E}^{\prime}$. White arrows point to trabeculae. a, atrium; v, ventricle. Scale bars: $50 \mu \mathrm{m}$ in $\mathrm{C}^{\prime}, \mathrm{E}^{\prime} ; 10 \mu \mathrm{m}$ in $\mathrm{C}^{\prime \prime}, \mathrm{E}^{\prime \prime}$

Here, we show that cardiac contraction is required for trabeculation through its role in initiating notch $1 b$ transcription in the ventricular endocardium. Active Notch1 signaling is detectable throughout the ventricular endocardium within $4 \mathrm{~h}$ of initiation of contraction and is restricted to the endocardial cushions during trabeculation. We further demonstrate that Notch1 activation induces the expression of its downstream effectors ephrin $b 2 a$ (efnb2a) and neuregulin 1 ( $n r g l)$ in the endocardium to regulate initiation of trabeculation. This endocardium-specific notch $1 b$ expression, and subsequent activation, requires functional primary cilia and the flow-responsive transcription factor Klf2a. Also, cultured endothelial cells respond to shear stress in a cilia-dependent manner to upregulate Notch1 and its downstream effectors, suggesting a role for primary cilia flow detection in endocardial Notch activation. Together, our findings suggest that in early cardiac morphogenesis, endocardial cells respond to cardiac contraction by detecting flow with primary cilia to regulate trabeculation by epistasis of notch $1 b$, efnb2a and $n r g 1$.

\section{RESULTS}

Cardiac contraction is required for myocardial trabeculation One of the earliest signs of cardiac chamber maturation is the formation of muscular, luminal protrusions called cardiac trabeculae. In zebrafish, cardiac trabeculae begin to form around 55 hours postfertilization (hpf) and are easily detectable at 3 days postfertilization (dpf) by examining cross-sections of ventricle outer curvature (Liu et al., 2010; Peshkovsky et al., 2011; Staudt et al., 2014). Although the basic structure of the heart can form in the absence of myocardial function, some morphogenic events require cardiac contraction. To determine whether cardiac contraction is required for trabeculation, we examined embryos deficient in troponin T type $2 a$ (cardiac) (tnnt $2 a$ ), which encodes an essential component of the contractile apparatus (Sehnert et al., 2002). We injected $\operatorname{Tg}($ myl $l: d s R e d) ; \operatorname{Tg}(k d r l: G F P)$ double transgenic embryos (labeling cardiomyocytes and endothelial cells, respectively) with standard control or tnnt $2 a$ morpholinos and assessed the presence of trabeculae at $3 \mathrm{dpf}$ (Fig. 1A). Like tnnt $2 a^{-1-}$ hearts, the noncontractile tnnt $2 a$ morphant hearts underwent relatively unaltered looping morphogenesis and chamber formation at $3 \mathrm{dpf}$, but notably, failed to form trabeculae (Chi et al., 2008; Staudt et al., 2014; Fig. 1B-C").

In order to determine whether failure to form trabeculae results from a direct role of Tnnt $2 \mathrm{a}$ or is more generally associated with contraction deficiency, we tested whether the trabeculation defects observed in tnnt $2 a$ morphants could be recapitulated by chemical inhibition of cardiac contraction. We thus treated $\operatorname{Tg}($ myl7:dsRed $)$; $T g(k d r l: G F P)$ embryos with vehicle or a pharmacological inhibitor of contraction from $22 \mathrm{hpf}$ to $3 \mathrm{dpf}$ (Fig. 1D). The hearts of embryos treated with blebbistatin, a myosin II ATPase inhibitor, were noncontractile on day 3 and strongly resembled tnnt $2 a$ morphants in that both myocardial and endocardial layers formed, but trabeculae were absent in the ventricle (Fig. 1E-E"). We further tested the effect of inhibition of cardiac contraction with verapamil, an L-type calcium channel blocker, and observed similar results (Fig. S1A-C'). Together, these data suggest that cardiac contraction is an important regulator of cardiac trabeculation.

\section{Cardiac contraction is required for endocardial Notch activation and notch $1 \mathrm{~b}$ transcription}

Cardiac contraction exerts mechanical forces on both myocardium and endocardium, and trabeculation requires crosstalk between these two layers (Tian and Morrisey, 2012; Wagner and Siddiqui, 2007). Thus, we next sought to determine whether any signaling pathways are activated in the ventricle in response to cardiac 

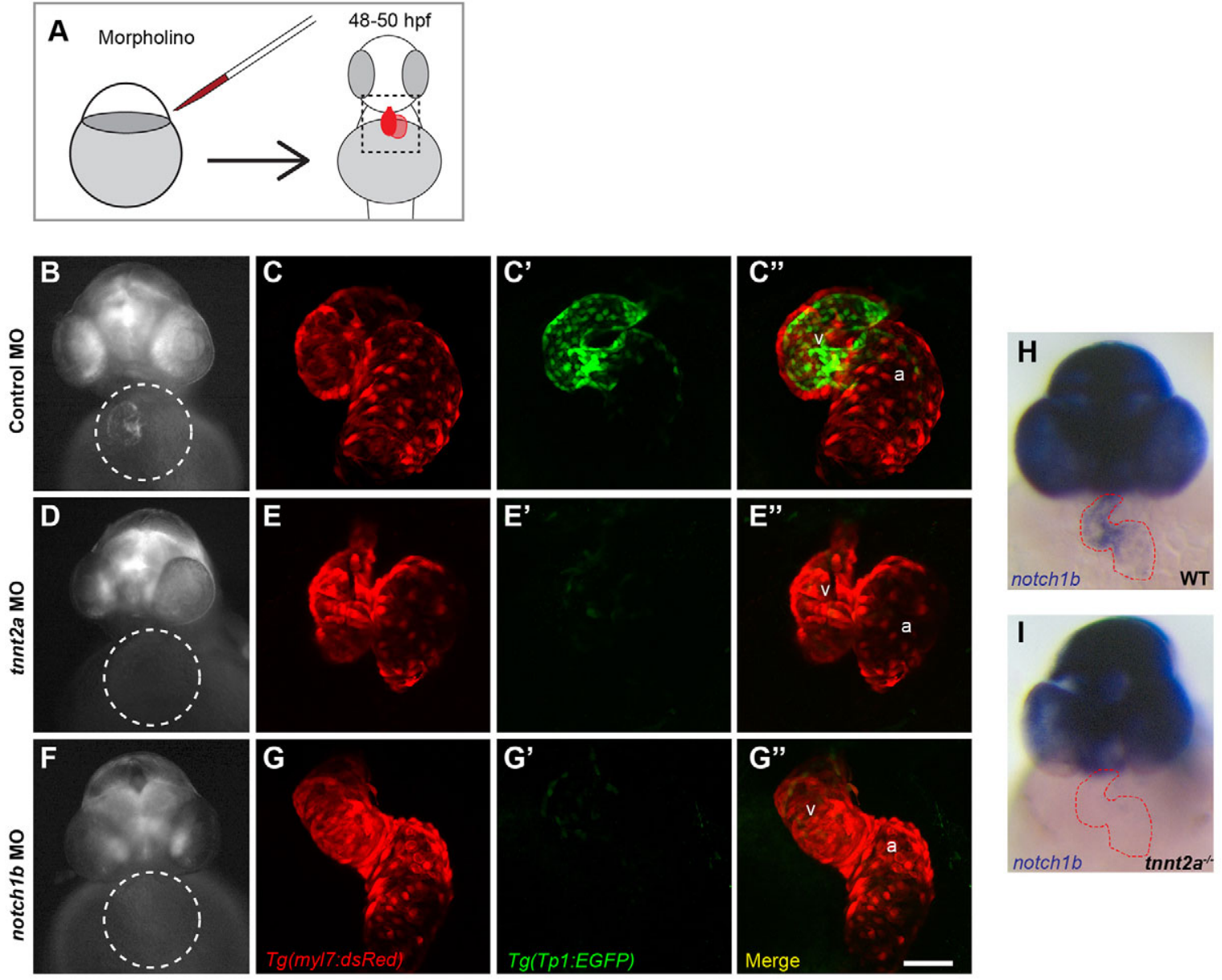

Fig. 2. Cardiac contraction is required for endocardial Notch activation and notch1b transcription. (A) Schematic diagram of morpholino (MO) gene knockdown experiment, in which double transgenic $T g(T p 1: E G F P) ; T g(m y l 7: d s R e d)$ embryos were injected with (B,C) control, (D,E) tnnt2a or (F,G) notch1b morpholinos and imaged at 48-50 hpf. (B,D,F) Representative whole-mount images of Notch reporter with cardiac regions highlighted by circles. (C,E,G) Confocal maximal intensity projections of the hearts shown in (B,D,F) with cardiomyocytes labeled in red, $\left(C^{\prime}, E^{\prime}, G^{\prime}\right)$ Notch reporters in green and $\left(C^{\prime \prime}, E^{\prime \prime}, G^{\prime \prime}\right)$ colocalized signal in yellow. Minimal colocalization indicates that Notch activation is in endocardial cells. (H,I) Whole-mount notch $1 b$ riboprobe hybridization in $(\mathrm{H})$ control and (I) tnnt $2 a^{-1-}$ embryos, with the heart outlined in red. Scale bar: $50 \mu \mathrm{m}$. a, atrium; v, ventricle.

contraction. Using $\operatorname{Tg}(T p 1: E G F P)$ Notch reporter embryos, in which the Notch1-responsive Tp1 module drives EGFP as a fluorescent readout for active Notch1 signaling (Parsons et al., 2009), we assessed Notch activation in control and tnnt $2 a$ morphants (Fig. 2A). At 48-50 hpf, Notch activation in control morphant hearts was robust in the ventricular endocardium and atrioventricular canal (AVC), with occasional weak signal detectable in the atrium (Fig. 2B-C"). Interestingly, Notch activation was below the limit of detection in tnnt $2 a$ morphant hearts of embryos examined in whole-mount or in confocal images (Fig. 2D-E"). By contrast, Notch signaling was robust in the brains of control and tnnt $2 a$ morphants, indicating a specific role for cardiac contraction in regulating endocardial Notch signaling (Fig. 2B,D). Similar results were observed with dimethyl sufoxide (DMSO)- and blebbistatin-treated embryos compared with control and tnnt $2 a$ morphants, respectively (Fig. S2A-C"). These data indicate that cardiac contraction is required for Notch activation in the endocardium at $2 \mathrm{dpf}$.

We next sought to identify the primary Notch receptor mediating Notch activation in the endocardium. Using a previously described morpholino to knock down notch1b (Milan et al., 2006; Wang et al., 2010) in $\operatorname{Tg}(T p 1: E G F P) ; \operatorname{Tg}($ myl7:dsRed $)$ double transgenic embryos, cardiac Notch activation was below detection in notch $1 b$ morphant hearts at 48-50 hpf (Fig. 2F-G"). To determine whether cardiac contraction regulates Notch1 activation by controlling notchlb gene transcription, we performed in situ hybridization at $48-50 \mathrm{hpf}$. notch $1 \mathrm{~b}$ expression mirrors $\operatorname{Tg}(\operatorname{Tp} 1$ : EGFP) Notch reporter expression in the heart at $48 \mathrm{hpf}$, but was below the limit of detection in tnnt $2 a^{-/-}$hearts (Fig. 2H,I). Thus, cardiac contraction controls Notch 1 signaling by regulating notch $1 b$ expression. Together, these findings indicate that cardiac contraction regulates notch $1 b$ at the transcript level in the heart. Our studies do not exclude the possibility that the mechanical forces associated with contraction might also regulate Notch receptor activation.

\section{Spatiotemporal pattern of Notch activation}

In order to gain a better understanding of how Notch activation might stimulate trabeculation, we characterized the spatiotemporal pattern of Notch activation in the developing zebrafish heart using the $\operatorname{Tg}(T p 1: V e n u s P e s t)$ Notch reporter transgenic line which expresses, under control of the Tp1 Notch response element, a partly destabilized fluorescent protein with a half-life of approximately $2 \mathrm{~h}$ compared with the $24 \mathrm{~h}$ half-life of GFP protein (Aulehla et al., 2008; Ninov et al., 2012). The use of this reagent afforded us greater spatial and temporal resolution to 
determine the time window of Notch activation in the ventricular endocardium. VenusPest expression was first detectable in the endocardium at $28 \mathrm{hpf}$, merely $4 \mathrm{~h}$ after initiation of contraction, with substantial spatial bias towards the end of the heart tube containing ventricular cardiomyocytes (Fig. 3A). VenusPest expression in the ventricular endocardium persisted until $55 \mathrm{hpf}$, after which it declined in parts of ventricular endocardium lining the outer curvature (Fig. 3B-D). By 72 hpf, VenusPest expression was primarily restricted to AVC endocardium, and was retained in the AVC until at least 4 weeks postfertilization (Fig. 3E,F; Fig. S3A). Thus, within the limits of reporter expression, Notch signaling is activated throughout the ventricular endocardium shortly after initiation of contraction, but becomes inactive shortly after the initiation of cardiac trabeculation and is completely restricted to the AVC by 3 dpf.

\section{Cardiac contraction promotes trabeculation through notch1b-efnb2a-nrg1 epistasis}

Our data demonstrate that, although Notch is required for trabeculation, it is not active in the ventricular endocardium during trabeculation, indicating that it controls trabeculation through intermediate signaling pathways. A recent report by Dietrich et al. (2014) demonstrated that blood flow and Bmp signaling regulate zebrafish endocardial chamber morphogenesis in a vascular endothelial growth factor (Vegf) signalingindependent manner. Interestingly, endocardial Notch activation and myocardial trabeculation appear to be independent of canonical Vegf signaling (Fig. S4). However, the arterial endothelial marker gene ephrin B2 (Efnb2) is required for trabeculation in mice directly downstream of the Notch1 transcriptional complex and upstream of neuregulin 1 ( Nrgl) (Grego-Bessa et al., 2007). We evaluated the role of these genes in cardiac trabeculation by assessing trabeculation at $3 \mathrm{dpf}$ in notch $1 b$ and efnb2a morphants. Using previously described splice-blocking morpholinos for notch $1 b$ and efnb2a (Milan et al., 2006; Wang et al., 2010), we knocked down their expression by at least $75 \%$ in the heart (Fig. S5A-D). At $3 \mathrm{dpf}$, notch $1 b$ and efnb2a morphants lacked trabeculae, whereas control morphants had trabeculae in the outer curvature (Fig. 4A-C'). To test further whether that Notch signaling is required for trabeculation in zebrafish, we examined mindbombl (mibl) mutants that are defective in Notch signaling because of a loss-of-function point mutation in the gene mindbomb E3 ubiquitin protein ligase 1. mib1 encodes an E3 ubiquitin ligase required for canonical trafficking of Notch ligands (Itoh et al., 2003). At $3 \mathrm{dpf}$, trabeculae were completely lacking in the ventricle of these mutant embryos (Fig. S6A,B).

To test whether efnb2a and $n r g 1$ could act downstream of Notch signaling to control cardiac trabeculation, and to establish the epistasis of these genes in zebrafish cardiac maturation, we assessed their expression in the zebrafish heart and found that notch $1 b$, efnb2a, nrg1 and their principal ligand or receptor partners are expressed at 48-50 hpf (Fig. S7). We isolated hearts at $48-50 \mathrm{hpf}$ and found that tnnt $2 a$ morphants had significant reduction in notch $1 b$, efnb2a and $n r g 1$ expression, whereas notch $1 b$ morphants had significant reduction in efnb2a and $n r g l$ transcripts levels (Fig. 4D,E). By contrast, notch $1 b$ expression and Notch activation pattern were not affected in efnb2a morphants, but $n r g 1$ expression was significantly reduced (Fig. 4F; Fig. S8). Notably, these defects in gene expression do not reflect failure of the endocardium to form, because both endocardium and myocardium are present at $3 \mathrm{dpf}$ (Fig. 4A-C'). Combined, these data suggest that cardiac contraction is required for trabeculation by activating a regulatory notch $1 b$ efnb2a-nrgl pathway.

Next, we asked whether forced activation of Notch signaling could bypass the requirement for cardiac contraction in trabeculation. To this end, we overexpressed the Notch intracellular domain to activate Notch signaling using the $\operatorname{Tg}$ (hsp 701:gal4); $\operatorname{Tg}(U A S: N I C D)$ system in control and tnnt $2 a$ morphants carrying the $\operatorname{Tg}(\operatorname{Tp} 1: E G F P)$ and $\operatorname{Tg}($ myl7:dsRed $)$ transgenes (Fig. 5A). All embryos were exposed to $37^{\circ} \mathrm{C}$ heat shock to activate Gal4 expression and, as an indication of forced Notch activation, we observed a dramatic increase in Tp 1:EGFP across all somatic tissues in approximately $25 \%$ of control and tnnt $2 a$ morphants at $48 \mathrm{hpf}$ (Fig. 5B). We isolated hearts from control and tnnt $2 a$ morphants and found significant upregulation of efnb2a and $n r g 1$ expression in tnnt $2 a$ morphant hearts with NICD
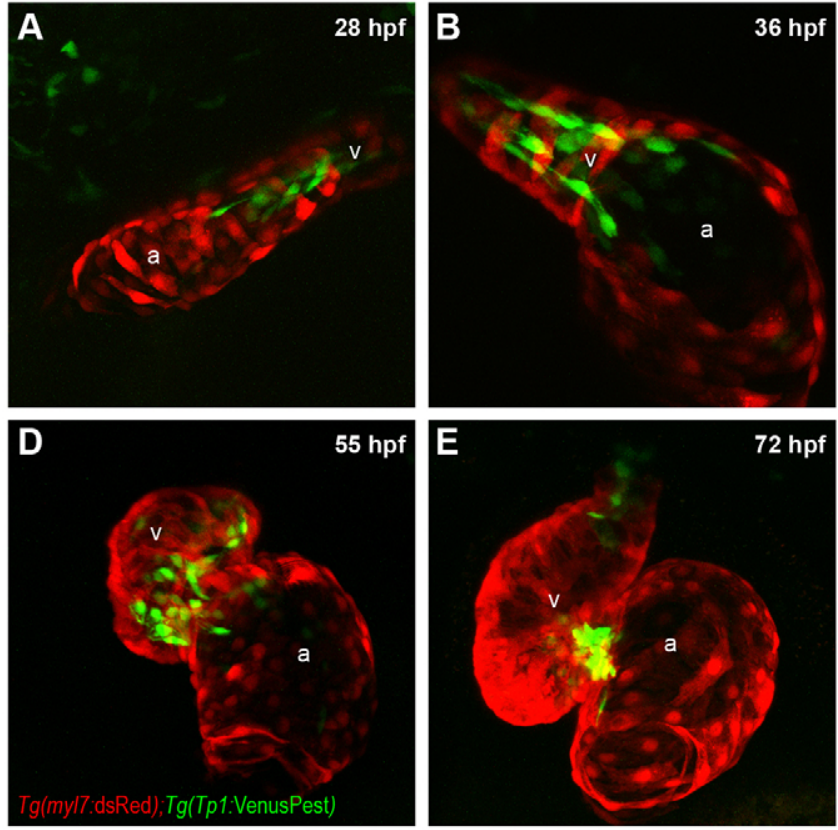
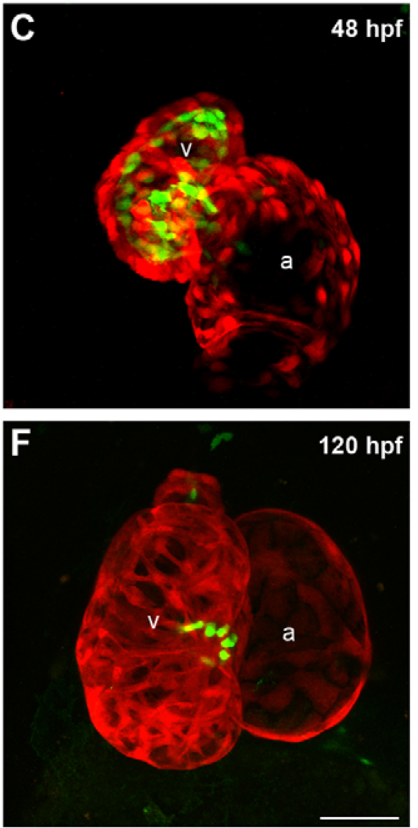

Fig. 3. Notch activation in the ventricular endocardium. (A-F) Confocal z-stack maximal intensity projection of hearts from double transgenic $T g(T p 1: V e n u s P e s t)$; $T g(m y / 7: d s R e d)$ embryos at designated time points, with Tp1:VenusPest expression in green and cardiomyocytes marked in red. Scale bar: $50 \mu \mathrm{m}$. a, atrium; v, ventricle. 

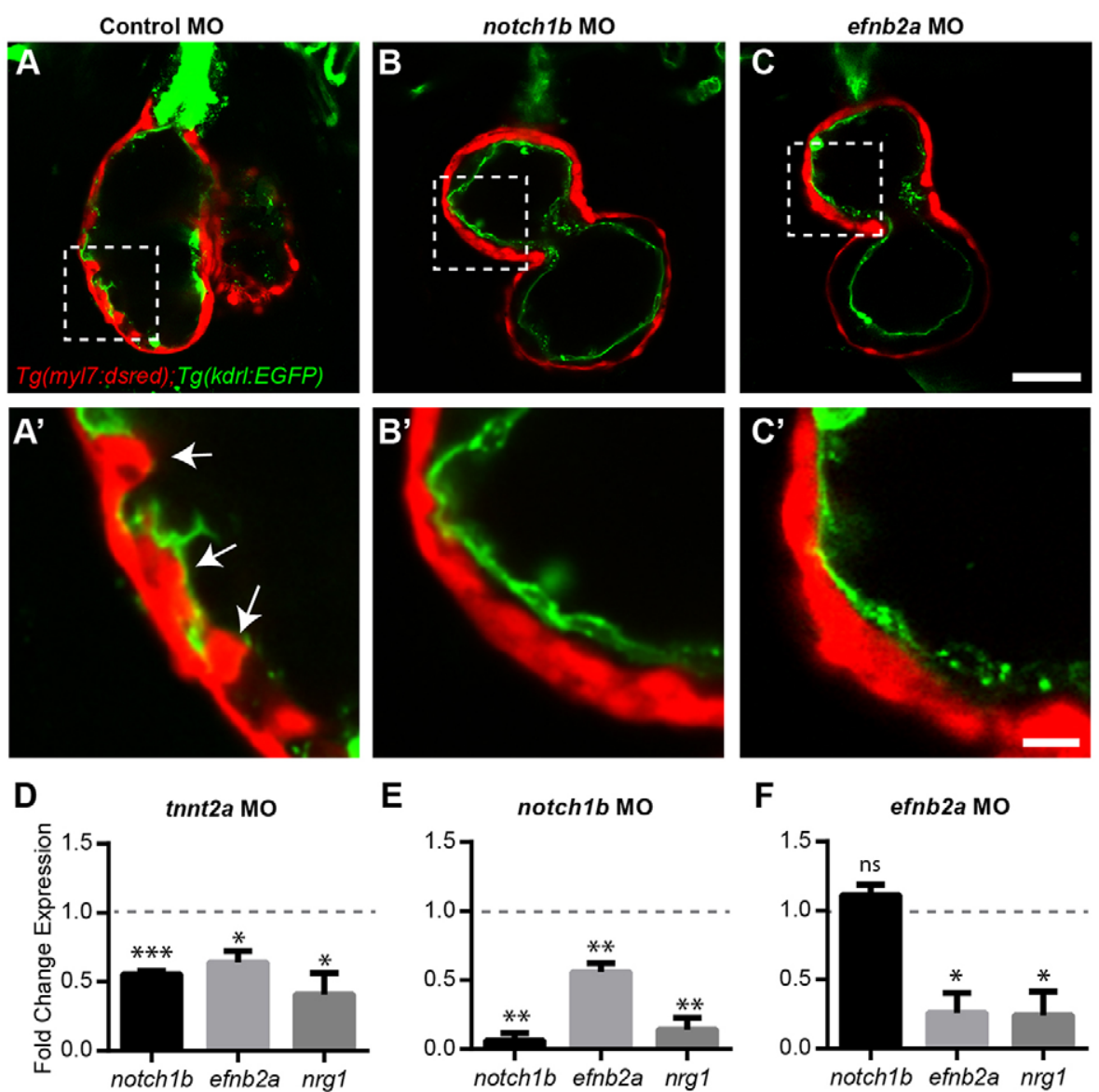

$\mathbf{F}$

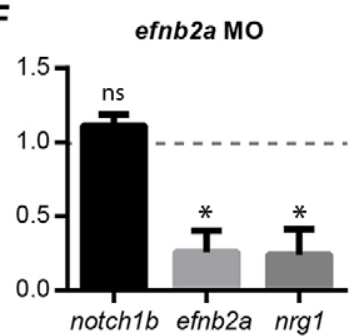

Fig. 4. Cardiac contraction promotes trabeculation through notch1b-efnb2a-nrg1 epistasis. $(A, B, C)$ Mid-chamber confocal optical section of $T g(m y l 7: d s R E D) ; T g(k d r l: E G F P)$ double transgenic (A) control, $(B)$ notch $1 b$ and $(C)$ efnb2a morphant (MO) hearts, showing cardiomyocytes in red and endocardial cells in green.

$\left(A^{\prime}, B^{\prime}, C^{\prime}\right)$ Magnified high-resolution images of the cardiac regions highlighted by dotted lines in $A, B$, C. (D-F) Expression of notch1b, efnb2a and $n r g 1$ in hearts isolated from (D) tnnt2a, (E) notch1b and (F) efnb2a morphants compared, normalized to expression in control morphant hearts (dashed line). ${ }^{*} P \leq 0.05-0.01,{ }^{*} P \leq 0.01-0.001,{ }^{* *} P<0.001$ compared with control morphants (one-sample t-test compared with control morpholino fold change=1). Error bars are s.e.m. White arrows point to trabeculae. Scale bars: $50 \mu \mathrm{m}$ in C; $10 \mu \mathrm{m}$ in $\mathrm{C}^{\prime}$ overexpression at 2-3 dpf (Fig. 5C,D). However, this upregulation was not sufficient to induce cardiac trabeculation in tnnt $2 a$ morphants (Fig. 5E-H').

\section{Primary cilia are required for Notch activation in endocardial cells}

We next asked how endocardial cells detect cardiac contraction to modulate Notch in the ventricle. Cardiac contraction exposes endocardial cells to mechanical forces across many different length and time scales (Bartman et al., 2004; Boselli et al., 2015; Lee et al., 2013; Vermot et al., 2009). Endocardial cells are particularly well positioned to detect hemodynamic forces, including shear stress (the frictional force of parallel flow). Endothelial primary cilia (microtubule-based sensory organelles that, in certain hemodynamic environments, protrude into the lumen of blood vessels) have a well-established role in detecting low levels of flow (Culver and Dickinson, 2010; Hahn and Schwartz, 2009; Slough et al., 2008; Van der Heiden et al., 2006). Recently, Goetz et al. (2014) demonstrated that endothelial primary cilia in the zebrafish vasculature are highly sensitive to low-magnitude shear forces from 24 to $28 \mathrm{hpf}$ to regulate vascular development. Thus, we hypothesized that primary cilia play a key role in flow detection, leading to notch $1 b$ upregulation and Notch1 activation in endocardial cells.

We validated the presence of primary cilia on endocardial cells around the time of Notch1 activation using Tg(actb2:Arl13b-GFP) transgenic embryos, where Arl13b-GFP localizes to primary cilia (Borovina et al., 2010). Although promoter activity for this transgene is higher in myocardial than endocardial cells, primary cilia can be detected in both cell layers (Fig. S9A,B). Given their potential role in flow detection, we focused on characterizing endocardial primary cilia. At $30 \mathrm{hpf}$, individual endocardial cells of non-injected embryos, control morphants and tnnt2 morphants possess a single, primary cilium projecting into the lumen of the heart (Fig. 6A-B'; Fig. S10A-B"). This indicates that endocardial cells possess primary cilia and that ciliation is independent of cardiac contraction.

Mutations in intraflagellar transport 88 homolog (ift88) cause ciliopathies in zebrafish (Kramer-Zucker et al., 2005; Lunt et al., 2009; Neugebauer and Yost, 2014; Tsujikawa and Malicki, 2004), and ift 88 morphants have deficiencies in endothelial primary cilia formation (Goetz et al., 2014). Likewise, we observed mislocalization of Arl13b-GFP in ift 88 morphants (arrowheads in Fig. S10A-C). To test whether endocardial primary cilia are involved in zebrafish ventricular endocardial Notch1 activation, we knocked down ift88 in $\operatorname{Tg}(T p 1: V e n u s P e s t) ; \operatorname{Tg}(m y l 7: d s R e d)$ double transgenic embryos and evaluated Notch1 activation in the morphants. Compared with control morphants, VenusPest expression was either undetectable or restricted to a few cells in the heart in cilia-deficient ift88 morphants (Fig. 6C-D'). Importantly, these morphants also exhibited reduced trabeculation (Fig. 6E-F'). Using images from whole-mount embryos, we compared mean fluorescence intensity of VenusPest in the ventricle and found a significant reduction in VenusPest expression in ift 88 morphants compared with control morphants (Fig. 6G). Additionally, we isolated hearts from ift 88 morphants and found a significant reduction in notch $1 b$ and $n r g l$ expression (Fig. 6H,I). 
A

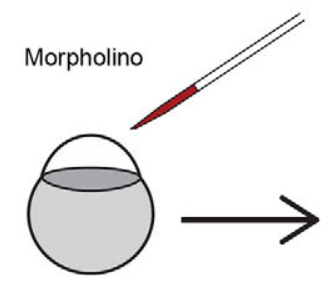

Tg(myl7:dsRed); $\operatorname{Tg}(T p 1: E G F P) ;$ Tg(hsp701:gal4);Tg(UAS:NICD)
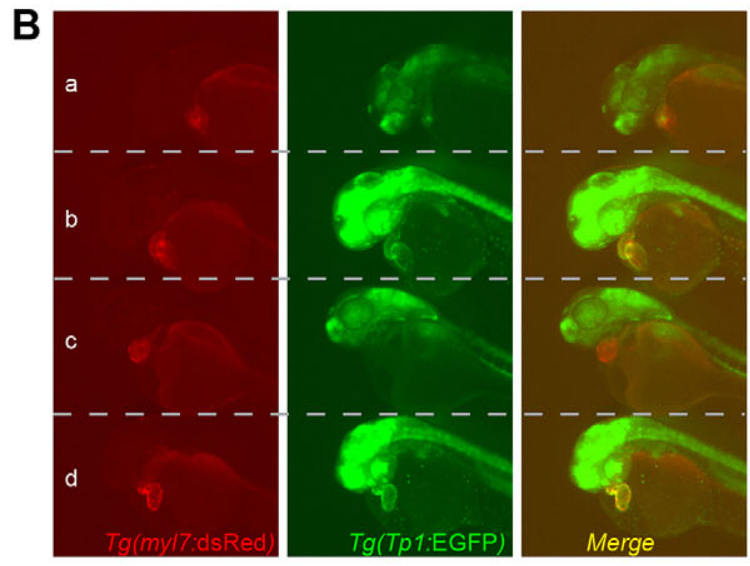

Control MO
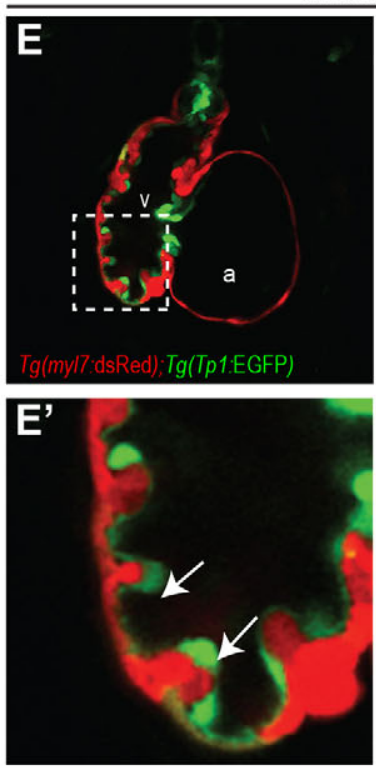

22-24hpf
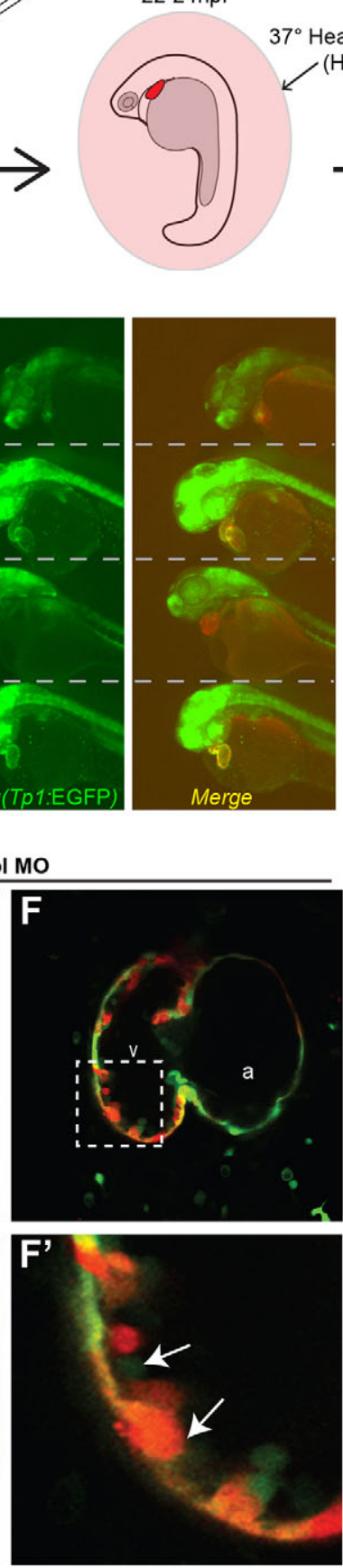

$37^{\circ}$ Heat Shock L (HS)

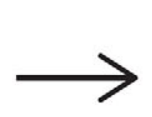

C

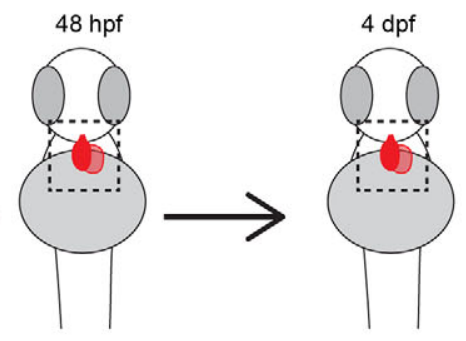

efnb2

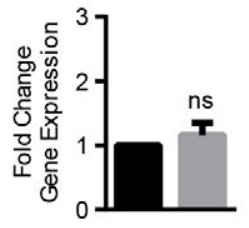

D

efnb2

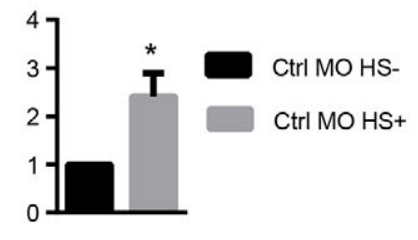

nrg1
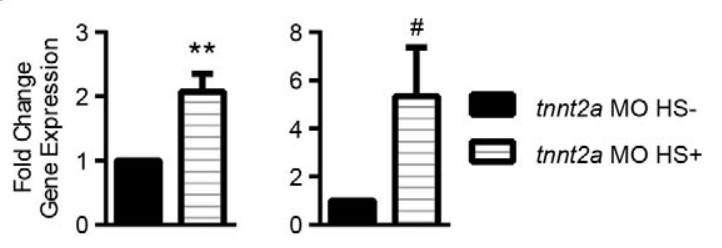

tnnt2a MO
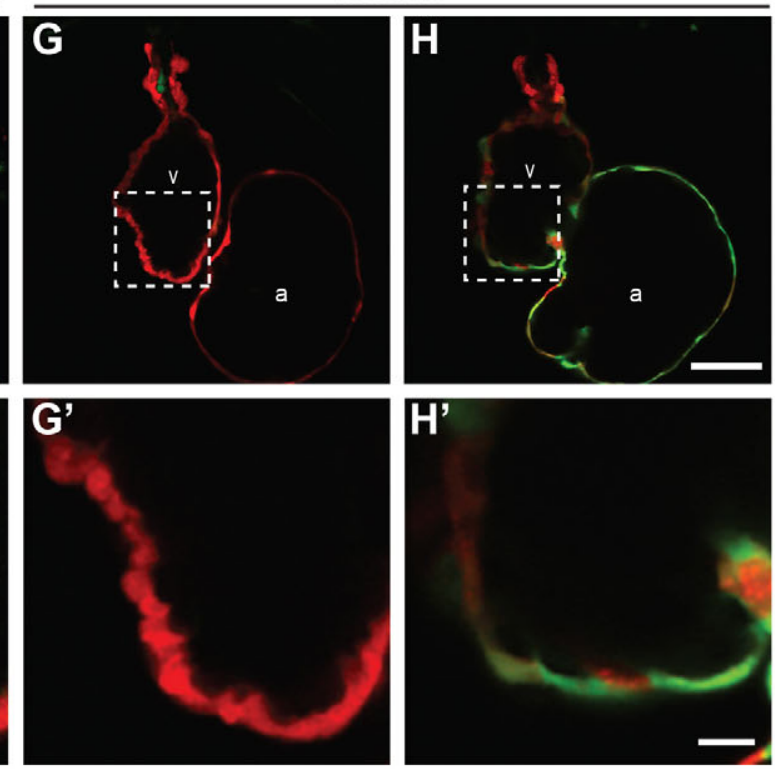

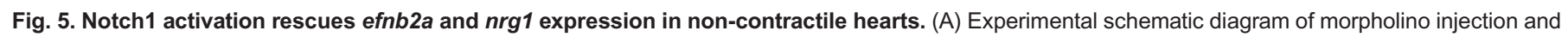
heat-shock overexpression of NICD. qRT-PCR and imaging were performed to examine gene expression and trabecular phenotype at 2-3 and 4 dpf, respectively. (B) Representative whole-mount images of cardiomyocytes (red) and Notch reporter (green) in (a,b) control and (c,d) tnnt2a morphants at 48 hpf (a,c) without or $(b, d)$ with NICD overexpression. (C,D) Expression of efnb2a and nrg1 in hearts isolated from (C) control morphants and (D) tnnt2a morphants, comparing gene expression in embryos with or without NICD overexpression. (E-H) Confocal mid-chamber optical section of $4 \mathrm{dpf}$ hearts, with dotted lines marking the inset magnified in $\mathrm{E}^{\prime}-\mathrm{H}^{\prime}$. ${ }^{\#} P \leq 0.075-0.05$, ${ }^{*} P \leq 0.05-0.01$, ${ }^{* *} P \leq 0.01-0.001$, ${ }^{* * *} P<0.001$ compared with control morphants (one-sample $t$-test compared with control

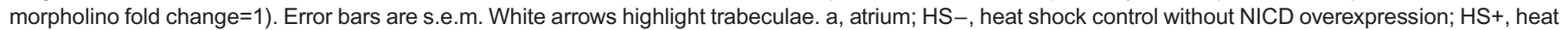
shock control with NICD overexpression; NICD, Notch intracellular domain; v, ventricle. Scale bars: $50 \mu \mathrm{m}$ in $\mathrm{H}$; $10 \mu \mathrm{m}$ in $\mathrm{H}^{\prime}$.

\section{Primary cilia are required at the onset of flow for Notch1 activity}

Although our data support a model in which primary cilia respond to luminal flow to activate Notch transcription, the loss of endocardial Notch activation in ift 88 morphants could be secondary to a role for primary cilia in early embryogenesis (Gerdes et al., 2009; Sasai and
Briscoe, 2012). To address this possibility, we used a pharmacological approach to define when primary cilia are necessary for endocardial Notch activation. Ciliobrevin D (CBD) inhibits the AAA+ ATPase motor cytoplasmic dynein and significantly reduces the microtubule cycling necessary to construct and maintain primary cilia (Firestone et al., 2012). We 

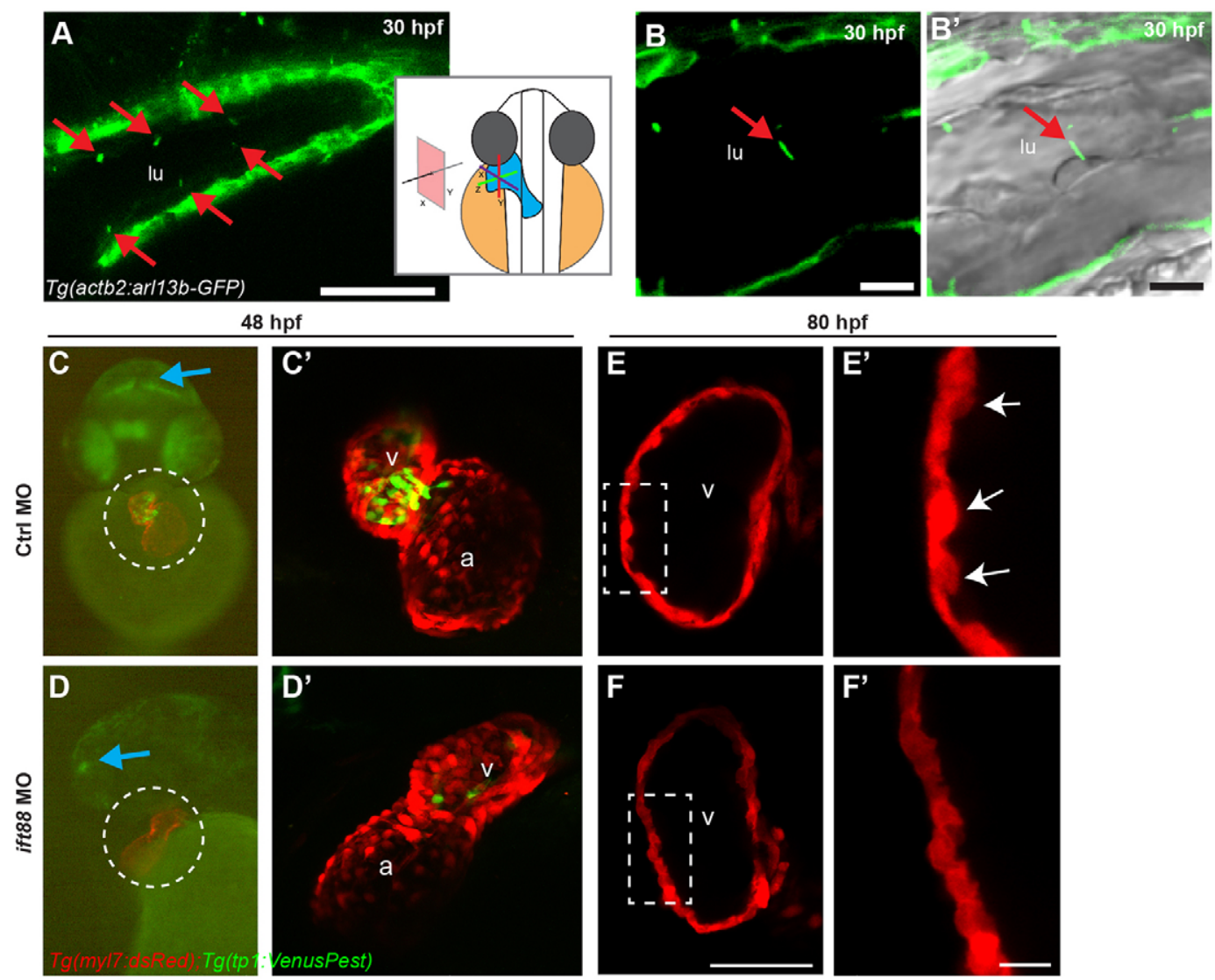

G

H
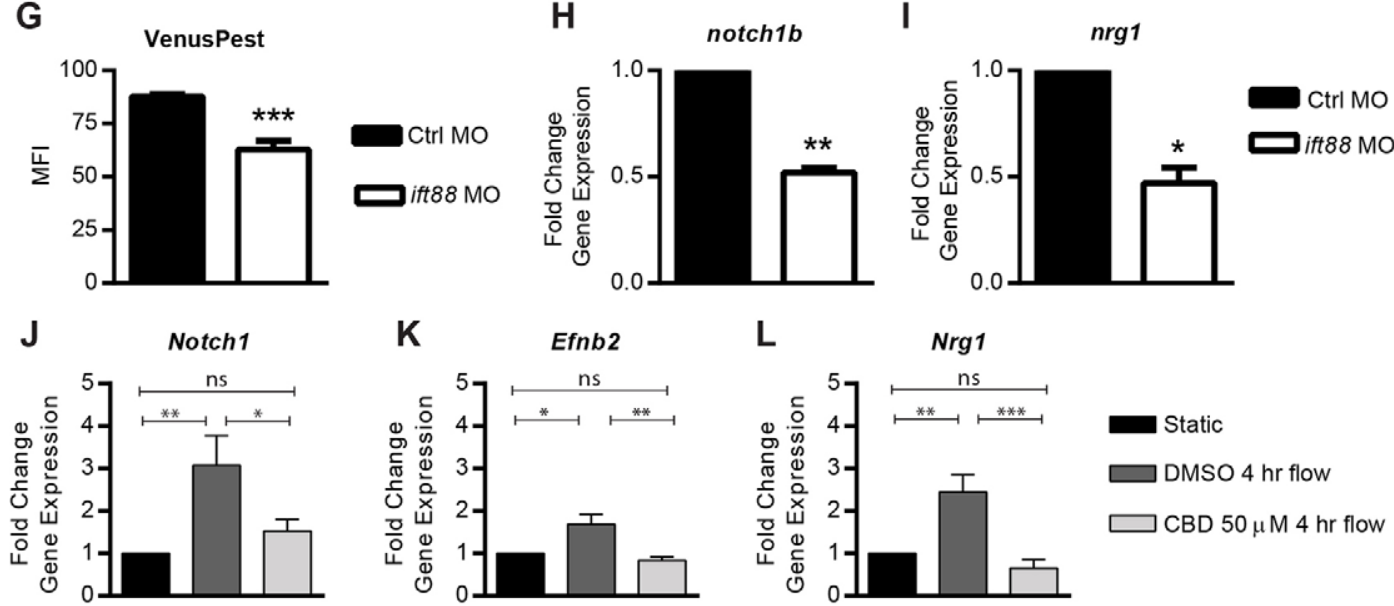

K
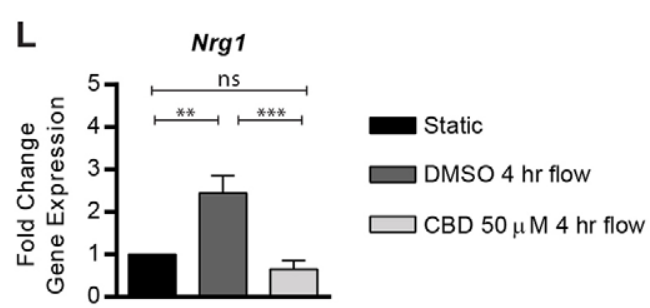

Fig. 6. Shear stress promotes notch1 expression in a primary cilia-dependent manner. (A-B') The hearts of $T g(a c t b 2: A r l 13 b-G F P)$ embryos were examined by confocal microscopy to assess localization of primary cilia in the endocardium. (A) $\operatorname{Tg}($ actb2:Arl13b-GFP) reporter expression in the heart. The schematic diagram indicates the orientation of the heart and confocal section relative to the whole embryo at $30 \mathrm{hpf}$. (B) High-resolution view of a $T g(a c t b 2: A r l 13 b-G F P)$ embryo merged with $\left(\mathrm{B}^{\prime}\right)$ a bright field image demonstrating colocalization of the primary cilium base with an endocardial cell. (C,D) Whole-mount $T g(m y l 7$ : dsRed); $\operatorname{Tg}\left(T p 1\right.$ :VenusPest) double transgenic (C) control and (D) ift88 morphants (MO) at $48 \mathrm{hpf}$. The hearts are marked with dashed circles. (C', $\left.\mathrm{D}^{\prime}\right)$ Confocal maximal projection of the heart from the individual embryos shown in C,D, overlaying cardiomyocytes (red) and Notch reporter (green). (E,F) Confocal optical section of the (E) control and (F) ift88 morphant embryo cardiomyocytes (red) at $80 \mathrm{hpf}$. The insets marked by the dotted rectangle were magnified in $\mathrm{E}^{\prime}, \mathrm{F}^{\prime}$.

(G) Quantification of ventricular Notch reporter (EGFP) MFI from whole-mount embryos at $48 \mathrm{hpf}$. (H,I) Relative expression of (H) notch1b and (I) nrg1 in control and ift88 morphant hearts. (J-L) Expression of (J) Notch1, (K) Efnb2 and (L) Nrg1 in DMSO- or $50 \mu \mathrm{M}$ CBD-treated MLECs that were exposed to 2 dyn/cm ${ }^{2}$ shear stress for $4 \mathrm{~h}$ compared with static DMSO- and CBD-treated controls. Red arrows highlight Arl13b-GFP in the endocardium. Blue arrows highlight Notch reporter signal in neural tissue. White arrows highlight trabeculae. ${ }^{*} P \leq 0.05-0.01,{ }^{* *} P \leq 0.01-0.001,{ }^{* * *} P<0.001$ compared with control morphants (one-sample $t$-test compared with 1.0-fold change or Student's $t$-test). Error bars are s.e.m. Scale bars: $50 \mu \mathrm{m}$ in A; $10 \mu \mathrm{m}$ in B, B', F'; $100 \mu \mathrm{m}$ in F. a, atrium; CBD, ciliobrevin D; lu, lumen; MFI, mean fluorescence intensity; v, ventricle.

treated $\operatorname{Tg}(T p 1:$ VenusPest $)$-positive embryos with DMSO or CBD starting at 18-24 hpf and assessed VenusPest expression at 42-48 hpf (Fig. S11A-D). DMSO injection at any of these times had no effect on VenusPest expression level (Fig. S11A,B,E). Embryos injected with CBD at 18 or $20 \mathrm{hpf}$ had reduced VenusPest expression (Fig. S11C,E), whereas embryos injected with CBD 
from 22 or $24 \mathrm{hpf}$ were indistinguishable from DMSO-injected controls (Fig. S11D,E). Given a time delay between initiation of treatment and sufficient accumulation for biological effects, these data suggest that Notch1 expression in the endocardium requires primary cilia in a short time window coincident with the onset of flow.

\section{Primary cilia probably detect low-magnitude shear stress to upregulate Notch in ventricular endocardial cells}

Primary cilia have two well-defined, independent functions: facilitating Hedgehog (Hh) signaling and detecting lowmagnitude shear stress (Anderson, 2006; Egorova et al., 2012; Goetz et al., 2014; Hierck et al., 2008; Roy, 2012; Van der Heiden et al., 2011; Wilson and Stainier, 2010). To determine whether Hh signaling is necessary for endocardial Notch activation, we treated $\operatorname{Tg}(T p 1: V e n u s P e s t) ; \operatorname{Tg}(m y l 7: d s R e d)$ Notch reporter embryos with cyclopamine to antagonize $\mathrm{Hh}$ signaling downstream of primary cilia (Chen et al., 2002; Stanton and Peng, 2010). Embryos treated with cyclopamine from 4 to $48 \mathrm{hpf}$ exhibited severe body axis deformities indicative of successful inhibition of Hh signaling (Fig. S12A,B). Embryos treated with cyclopamine from 18 to $48 \mathrm{hpf}$ and 24 to $48 \mathrm{hpf}$ did not exhibit defects in Notch activation (Fig. S12C-J). This argues against a role of canonical Hh signaling in controlling endocardial Notch activation, and implicates primary cilia flow detection in endocardial Notch activation and cardiac trabeculation.

Flow magnitude and directionality influence the biological responses elicited in endothelial cells. Although our data indicate that flow is important for activating Notch, the differential roles of shear stress magnitude and flow directionality are unclear. In zebrafish, shear stress and retrograde flow at $2 \mathrm{dpf}$ can be manipulated through knockdown of gatala and gata2a, two transcription factor genes important for hematopoiesis (Vermot et al., 2009). Interestingly, reducing hematocrit to reduce shear stress and altering retrograde flow fraction in this manner lead to neither reduced Notch reporter expression at $48 \mathrm{hpf}$ nor loss of trabeculation by $80 \mathrm{hpf}$ (Fig. S13A-M). Increasing the retrograde flow fraction by knocking down gata2a, with or without coknockdown of gatala, slightly increased Notch activation in the ventricle, although reduced ventricular size could also contribute to this phenotype (Fig. S13E,F). Given that we see Notch activation as early as $28 \mathrm{hpf}$, which is before the hematocrit comprises a large fraction of blood volume, we suggest that these results support a role for low levels of flow in Notch activation and cardiac trabeculation.

As contraction and flow cannot be decoupled in the developing zebrafish ventricle by existing genetic means, we used an in vitro model of shear stress to assess the role of primary cilia shear stress detection in regulating Notchl expression directly. We used immortalized mouse embryonic endothelial cells (MLECs), which are known to upregulate Notch pathway genes in response to shear stress, to model endocardial cilia-dependent flow responses (Sweet et al., 2013). MLECs were pretreated with either DMSO as vehicle control or CBD to inhibit formation of primary cilia and exposed for 0 or $4 \mathrm{~h}$ to low-magnitude shear stress $\left(<2 \mathrm{dyn} / \mathrm{cm}^{2}\right)$, comparable to the fluid forces exposed to endocardial cells in early heart development (Goetz et al., 2014). We observed significant elevation in Notch1, Efnb2 and Nrg1 in DMSO-treated MLECs compared with static controls. Interestingly, this upregulation was inhibited by CBD treatment (Fig. 6J-L). MLECs pretreated with either DMSO or CBD demonstrated dynamic regulation of the flowresponse gene Kruppel-like factor 2 (Klf2), suggesting that some non-cilia-based flow-detection mechanisms are not perturbed by

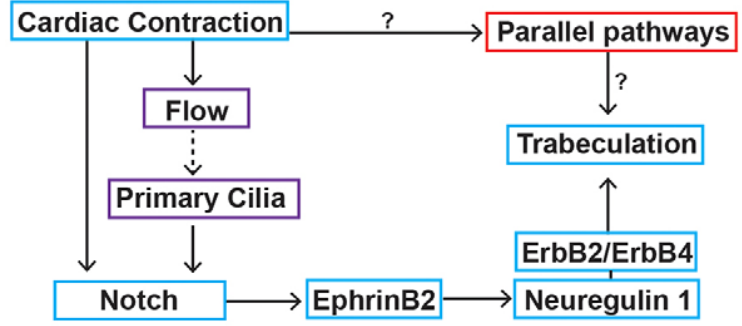

Fig. 7. Cardiac contraction activates endocardial Notch signaling in a primary cilia-dependent manner to regulate trabeculation. Schematic diagram. (Blue boxes) Cardiac contraction activates a regulatory Notch-Ephrin B2a-Neuregulin 1 pathway in endocardial cells to activate ErbB2 signaling in cardiomyocytes to promote trabeculation. (Red box) Given that Notch activation in non-contractile hearts is not sufficient for trabeculation, cardiac contraction also stimulates parallel pathways to promote trabeculation. (Purple boxes) Cardiac contraction causes blood flow, which is likely to be detected by primary cilia to activate Notch1.

CBD treatment (Fig. S14). Interestingly, $k l f 2 a$ was also required for endocardial Notch activation and trabeculation (Fig. S15). Thus, shear stress stimulates endothelial cells to increase expression of Notch1, Efnb2 and Nrgl in a myocardium-independent manner. Combined, these data indicate that primary cilia are important mediators of shear stress to regulate endocardial Notch signaling prior to trabeculation.

Together, our data support a model in which cardiac contraction initiates flow, which is detected by primary cilia on endocardial cells to activate a regulatory notch1-efnb2a-nrgl pathway and promote cardiac trabeculation (Fig. 7).

\section{DISCUSSION}

Trabeculation, the formation of muscular protrusions that increase myocardial mass prior to coronary vascularization, is an essential aspect of ventricular maturation (Liu et al., 2010; Moorman and Christoffels, 2003; Samsa et al., 2013; Sedmera et al., 2000). Although previous work clearly demonstrated the utility of zebrafish embryo in studying cardiac morphogenesis, mechanistic detail has been lacking to describe how mechanical forces guide the cellular changes that execute heart chamber maturation in zebrafish. In the present study, we present evidence supporting a model in which flow, caused by cardiac contraction, is detected by primary cilia on endocardial cells to stimulate notchlb expression and regulate trabeculation through notch $1 b-e f n b 2 a-n r g 1$ epistasis. Thus, our study reveals a molecular mechanism that links flow sensing and cell signaling to ventricular maturation in the developing heart.

Our previous work demonstrated that ErbB2 is required to initiate trabeculation in the heart at $2 \mathrm{dpf}$ (Liu et al., 2010). Examining this phenotype further, we found that eliminating cardiac contraction by injection of tnnt $2 a$ morpholino prevented trabeculation and endocardial Notch activation but did not affect erbb2 expression levels in the heart. Thus, we hypothesized that cardiac contraction controls expression of the erbb2-erbb4 ligand, $n r g 1$, to modulate trabeculation. Previous studies in the mouse indicate a crucial role of an endocardial Notch1-Efnb2-Nrg1 regulatory pathway in cardiac trabecular formation (Grego-Bessa et al., 2007). We asked whether these genes are required downstream of cardiac contraction for zebrafish cardiac trabeculation. Indeed, notch $1 b$ and efnb $2 a$ morphants lacked cardiac trabeculae at $3 \mathrm{dpf}$, and gene expression analysis confirmed epistasis of notchlb-efnb2-nrgl with respect to cardiac contraction. Although the potential off-target effects of morpholinos could limit our interpretation of the morphant 
phenotypes, the fact that notch $1 b$ morphants and mibl mutants exhibit a similar trabecular defect supports a role of Notch signaling in cardiac trabeculation. In addition, our data are also supported by the work from the de la Pompa group showing that Efnb2 acts directly downstream of Notch1 to regulate trabeculation (GregoBessa et al., 2007). In addition to the trabeculation defect, we noticed separation of the endocardium and myocardium. This separation is also evident in sections and scanning electron micrographs of mouse embryos with genetic deletion of Notch1, Efnb2, Nrg1 and Bmp10 (Grego-Bessa et al., 2007). Whether this is because of failure of the cardiac jelly to degrade or detachment of the endocardium remains to be determined, but it appears to be a defect common to many trabeculation phenotypes.

Notch signaling plays many roles in heart development. In zebrafish, we had the opportunity to observe the pattern of Notch activation directly with fine spatial and temporal resolution. The highly stereotyped spatiotemporal distribution of Notch signaling that we observed (Fig. 3) is interesting for several reasons. First, although our data reaffirm the importance of Notch in vertebrate trabeculation, our study indicates a somewhat different pattern of Notch activation in zebrafish heart development. In zebrafish, Notch is active shortly after initiation of blood flow and is not active in the ventricular endocardium during trabeculation. In mice, the NICD is localized in the nucleus of endocardial cells from E8.5 to E9.5 during the first stages of trabeculation, and subsequently is localized preferentially in the nuclei of endocardial cells at the base of trabeculae (Grego-Bessa et al., 2007). This might reflect differential roles of Notch signaling in zebrafish versus mouse heart development, possibly because of differences in heart size and timing of major morphogenic processes. Second, the spatial segregation of Notch activation between ventricle, atrium and AVC endocardium from 1 to $3 \mathrm{dpf}$ suggests that there are previously underappreciated, underlying differences between endocardial cells in these spatially distinct regions, leading to different roles in the maturing heart. Whether their difference in Notch activation are intrinsic properties or result from differential cues derived from the overlying myocardium remains to be seen.

In the present study, we also provided evidence suggesting that functional primary cilia are required at the onset of flow for notch $1 b$ expression in the ventricular endocardium of the developing heart. This expression is important for Notch activation and cardiac trabeculation. Cardiac contraction and blood flow are intimately associated and cannot be decoupled easily in the developing zebrafish ventricle by genetic means. Although endocardial cells might arise from either vascular endothelial cells or cardiac progenitors, they are a type of endothelial cell and are highly sensitive to flow (Bussmann et al., 2007; Kattman et al., 2006; Milgrom-Hoffman et al., 2011; Moretti et al., 2006; Vermot et al., 2009). We used an in vitro system to apply low-magnitude flow to endothelial cells to model endocardial, cilia-dependent flow responses and found that flow stimulated Notch1, Efnb2 and Nrg1 expression in the absence of myocardially derived factors or circulating factors in the bloodstream. This upregulation was blocked by pretreating cells with CBD, a small molecule which inhibits the dynein motor protein responsible for trafficking microtubules to primary cilia (Firestone et al., 2012). Notably, although CBD treatment reduces the size and number of primary cilia, it also regulates mitotic spindle formation and organelle transport (Firestone et al., 2012). Thus, our interpretation does not account for the possibility that our observed effects might result from non-specific effects of CBD.

Although our data showed that a notch1b-efnb2a-nrg1 pathway is required for cardiac chamber maturation, it is not the only genetic network activated by cardiac contraction and involved in trabeculation. Trabeculation is a complex morphogenic event that requires precise coordination of molecular and cellular events in both myocardial and endocardial cells. We found that, although forced NICD expression was sufficient to upregulate efnb2a and $n r g 1$ in tnnt $2 a$ morphants, it did not induce trabeculation. Curiously, it was also insufficient to rescue trabeculation in ift 88 morphants (our unpublished data). Given that NICD was overexpressed in all tissues, we suspect that the lack of trabeculation might be because of an inhibitory role of myocardial overactivated Notch1 signaling in cardiac trabeculation and a loss of other primary cilia downstream signaling. It will be interesting to use separate myocardial and endocardial Gal4 lines to investigate the precise spatiotemporal role of Notch activation in regulating chamber morphogenesis. Additionally, deficiencies in the luminal protrusions extended by cardiomyocytes during trabeculation, which are unstable in tnnt $2 a$ morphants, could limit trabeculation in tnnt2a morphants (Staudt et al., 2014).

Our work prompts many important questions about primary cilia and the role of hemodynamics in cardiac morphogenesis. Much of the work on the role of hemodynamics in endocardial morphogenesis has focused on valvulogenesis. Owing to its position in the heart, the AVC is exposed to an extremely dynamic flow environment in the embryo. Given that primary cilia disassemble in high-shear-stress environments (Iomini et al., 2004) and Notch activity becomes restricted to the AVC after $3 \mathrm{dpf}$, it will be interesting to explore the differential role of primary cilia for Notch activation in AVC and ventricular endocardium. Others have found that modifying the hemodynamic environment in the heart can alter the position of trabeculae, suggesting that other mechanosensors might be involved in later cardiac morphogenesis (Peshkovsky et al., 2011). Interestingly, the flow response gene klf2a is upstream of notchlb (Vermot et al., 2009) and is required for Notch activation and cardiac trabeculation. However, neither lowering the magnitude of shear stress nor altering the retrograde flow fraction using gatala, gata2a or gatala/2a morpholinos, as described by Vermot et al. (2009), was capable of preventing endocardial Notch activation or trabeculation; thus, much work remains to be done in order to define the precise hemodynamic cues involved in endocardial Notch activation.

Our work, combined with a recent study showing that primary cilia bending in response to blood flow is a major regulator of vascular development from 24 to $28 \mathrm{hpf}$ (Goetz et al., 2014), strongly suggest that primary cilia are involved in sensing lowmagnitude shear stress in vivo to regulate cardiovascular morphogenesis. The precise mechanism by which primary cilia detect flow is an area of active research. One theory suggests that stretch-activated transient receptor potential (TRP) channels coupled to primary cilia at the membrane are activated when primary cilia bend, leading to increased intracellular calcium and intracellular signaling (Delling et al., 2013; Goetz et al., 2014; Yoshiba et al., 2012). Interestingly, mutations in the mechanosensitive TRP family result in major valve defects, and TRP channel activity appears to depend on an oscillatory flow pattern (Heckel et al., 2015).

Overall, our data support a model in which endocardial primary cilia are the physical mechanism by which endothelial cells detect low flow to stimulate endocardial notchlb expression in the zebrafish heart to promote ventricular chamber maturation. Future studies are needed to determine the precise role of primary cilia in detecting flow in the developing heart. 


\section{MATERIALS AND METHODS}

\section{Zebrafish husbandry and stocks}

All animals were maintained at the aquaculture facility of the University of North Carolina at Chapel Hill in accordance with Institutional Animal Care and Use Committee approved protocols (Westerfield, 2000). The zebrafish lines used in this study are as follows: tnnt $2 a^{b 109}$ (Sehnert et al., 2002), $m^{m i b^{t a 52 b}}$ (Itoh et al., 2003; Jiang et al., 1996; van Eeden et al., 1996), $\operatorname{Tg}(m y l 7: G F P)^{t w u 26}$ (Huang et al., 2003), Tg(myl7:dsRed) ${ }^{v c 6}$ (Rothschild et al., 2009), $\operatorname{Tg}(m y l 7: m k a t e C A A X)^{\text {sdl1 }}$ (Lin et al., 2012), $\operatorname{Tg}(\mathrm{kdrl}$ : $E G F P)^{s 843}$ (Jin et al., 2005), $T g(k d r l: m C h e r r y)^{s 896}$ (Bertrand et al., 2010), $\operatorname{Tg}(T p 1: E G F P)^{u m 14}$ (Parsons et al., 2009), Tg(Tp1:VenusPest) ${ }^{s 940}$ (Ninov et al., 2012), $\operatorname{Tg}(-1.5 h s p 70 l: G a l 4)^{k c a 4}$ (Scheer and Campos-Ortega, 1999), $\operatorname{Tg}\left(\right.$ UAS:notch 1a-intra ICD) ${ }^{k c a 3}$ (Scheer et al., 2001) and Tg(actb2:Arll3b$G F P)^{h s c 5 T g}$ (Borovina et al., 2010). In all studies, embryos were maintained at $28.5^{\circ} \mathrm{C}$ in either embryo water or system water and treated continuously with $0.003 \%$ 1-phenyl 2-thoiurea starting at 20-24 hpf.

\section{Morpholino injections}

Morpholino oligonucleotides (see Table S1) were diluted in 5 mM HEPES containing $0.05 \%$ Phenol Red, and $1 \mathrm{nl}$ was injected into a minimum of 100 embryos at the one-cell stage.

\section{Drug treatments}

For pharmacological inhibitor studies, dechorinated embryos were treated in $4 \mathrm{ml}$ of $6 \mu \mathrm{M}$ blebbistatin (Cell Signaling) in embryo water or $200 \mu \mathrm{g} / \mathrm{ml}$ verapamil (Sigma) in 1\% DMSO-containing embryo medium $(5 \mathrm{mM} \mathrm{NaCl}$, $0.17 \mathrm{mM} \mathrm{KCl}, 0.33 \mathrm{mM} \mathrm{CaCl}_{2}$ and $0.33 \mathrm{mM} \mathrm{MgSO}_{4}, \mathrm{pH}$ 6.8-6.9). Further details of embryo drug treatments are given in the supplementary Materials and Methods.

\section{Imaging and processing}

Anesthetized embryos were mounted in low-melt agarose (Sigma) in embryo medium and manually oriented for optimal visual access to the heart. Epifluorescence images were collected on a Leica M205C fluorescence stereoscope. Data were collected for a minimum of 10 embryos and quantified using ImageJ (Schneider et al., 2012). For confocal images, embryos were sacrificed and the top $75 \%$ of the heart was imaged using a Olympus Fluoview 1000MPE microscope. Confocal data were collected for a minimum of three embryos for each condition representative of pool of a minimum of $n>12$ embryos that were visually inspected for phenotype. For further details of microscopy, image collection and analysis parameters please refer to the supplementary Materials and Methods.

\section{In situ hybridization}

In situ hybridization was performed as previously described (Liu and Stainier, 2010). The in situ hybridization probe for notch $1 b$ was prepared as previously described (Milan et al., 2006) and synthesized from pGEMT vector (Promega) using the DIG RNA labeling kit (Roche). Whole-mount embryo imaging was performed on a Leica MZ16F fluorescence stereomicroscope.

\section{Heart isolation}

Hearts were manually isolated from embryos sacrificed at 48-72 hpf using a Leica M205C fluorescence stereomicroscope and visualizing cardiac tissue using $\operatorname{Tg}(m y l 7: E G F P)$ signal, then trimmed free of non-cardiac tissue and transferred to ice-cold lysis buffer. Tissue was homogenized, and RNA was isolated from the homogenate using the RNAeasy Micro kit (Qiagen). Single-strand complementary DNA synthesis was performed on freshly isolated RNA using the Bio-Rad iScript cDNA synthesis kit. At least ten hearts were pooled for each condition.

\section{Cell culture}

MLECs were prepared as described previously (Sweet et al., 2012). MLECs at passage 38-45 were cultured on gelatin-coated plastic tissue culture plates and maintained in DMEM (Gibco, 11995), 10\% fetal bovine serum (Sigma), 1\% penicillin-streptomycin (Gibco), 1\% non-essential amino acids (Gibco) and $0.009 \% \beta$-mercaptoethanol.

\section{In vitro shear stress}

Confluent, cobblestone-stage MLECs were starved for 12-16 h in culture media (described in the previous subsection) containing $1 \%$ fetal bovine serum, then pretreated with DMSO or $50 \mu \mathrm{M}$ ciliobrevin D (Millipore) for $2 \mathrm{~h}$ before initiation of flow. Shear stress, $2 \mathrm{dyn} / \mathrm{cm}^{2}$, was generated using a previously described cone and plate viscometer (Sorescu et al., 2003). After 0,1 or $4 \mathrm{~h}$ exposure to shear stress, cells were washed with ice-cold PBS then lysed and RNA was extracted using Trizol (Invitrogen) according to manufacturer's instructions. RNA was reverse transcribed using Superscript III First-Strand Synthesis Supermix (Invitrogen) and gene expression assessed as described below.

\section{Expression analysis}

For qRT-PCR, the National Center for Biotechnology Information's PrimerBLAST was used to design exon-spanning, gene-specific SYBRGreen primers. All primers were validated by high-resolution melt analysis, size confirmation, RNA-only and no-template controls. See Table S2 for primer sequences. SybrGreen real-time PCR was performed in triplicate using Viia7 real-time PCR system (Invitrogen). For quantification, we used the $\Delta \Delta C_{\mathrm{t}}$ method, in which raw $C_{\mathrm{t}}$ values were normalized to $a c t b$ as a housekeeping gene and appropriate baseline condition, then fold change calculated as $2^{-\Delta \Delta C t}$

\section{Statistical analysis}

Values are presented as means \pm s.e.m. Statistical significance was determined by one-sample $t$-test (between one group and a reference value) or Student's $t$-test (between two groups).

\section{Acknowledgements}

We thank Drs Vicki Bautch, Nathan Lawson and Brian Ciruna for fish stocks. We also thank the University of North Carolina Olympus Imaging Research Center for confocal microscope use, and the University of North Carolina Zebrafish Aquaculture Core Facility for fish care and microscope use. We thank David Staudt for discussion during the early stages of this work.

\section{Competing interests}

The authors declare no competing or financial interests.

\section{Author contributions}

L.A.S. designed and performed the majority of experiments, analyzed data and wrote the manuscript. C.G. designed and assisted with the in vitro flow experiment. E.T., D.Y.R.S. and L.Q. provided intellectual input and supervised the work. J.L. designed and performed experiments, analyzed data and wrote the manuscript. All authors commented on the manuscript.

\section{Funding}

L.A.S. and C.G. are supported by National Institutes of Health T32 grant [HL06976814; PI, Nobuyo Maeda]. This study was supported by National Institutes of Health/ National Heart, Lung, and Blood Institute [R00 HL109079 grant] and American Heart Association [grant 15GRNT25530005 to J.L.], National Institutes of Health/National Heart, Lung, and Blood Institute [1R01HL117256 grant to E.T.], National Institutes of Health/National Heart, Lung, and Blood Institute [1RO1HL54737 to D.Y.R.S.], the American Heart Association Scientist Development Grant [13SDG17060010 to L.Q.] and the Ellison Medical Foundation New Scholar Grant [AG-NS-1064-13 to L.Q.]. Deposited in PMC for release after 12 months.

\section{Supplementary information}

Supplementary information available online at

http://dev.biologists.org/lookup/suppl/doi:10.1242/dev.125724/-/DC1

\section{References}

Anderson, K. V. (2006). Cilia and Hedgehog signaling in the mouse embryo. Harvey Lect. 102, 103-115.

Aulehla, A., Wiegraebe, W., Baubet, V., Wahl, M. B., Deng, C., Taketo, M., Lewandoski, M. and Pourquié, O. (2008). A beta-catenin gradient links the clock and wavefront systems in mouse embryo segmentation. Nat. Cell Biol. 10 186-193.

Auman, H. J., Coleman, H., Riley, H. E., Olale, F., Tsai, H.-J. and Yelon, D. (2007) Functional modulation of cardiac form through regionally confined cell shape changes. PLOS Biol. 5, e53.

Bartman, T., Walsh, E. C., Wen, K.-K., McKane, M., Ren, J., Alexander, J., Rubenstein, P. A. and Stainier, D. Y. (2004). Early myocardial function affects endocardial cushion development in zebrafish. PLoS Biol. 2, e129. 
Benedito, R. and Hellström, M. (2013). Notch as a hub for signaling in angiogenesis. Exp. Cell Res. 319, 1281-1288.

Bertrand, J. Y., Chi, N. C., Santoso, B., Teng, S., Stainier, D. Y. R. and Traver, D. (2010). Haematopoietic stem cells derive directly from aortic endothelium during development. Nature 464, 108-111.

Borovina, A., Superina, S., Voskas, D. and Ciruna, B. (2010). Vangl2 directs the posterior tilting and asymmetric localization of motile primary cilia. Nat. Cell Biol. 12, 407-412.

Boselli, F., Freund, J. B. and Vermot, J. (2015). Blood flow mechanics in cardiovascular development. Cell. Mol. Life Sci. 72, 2545-2559.

Bussmann, J., Bakkers, J. and Schulte-Merker, S. (2007). Early endocardial morphogenesis requires Scl/Tal1. PLoS Genet. 3, e140.

Chen, J. K., Taipale, J., Cooper, M. K. and Beachy, P. A. (2002). Inhibition of Hedgehog signaling by direct binding of cyclopamine to Smoothened. Genes Dev. 16, 2743-2748.

Chi, N. C., Shaw, R. M., Jungblut, B., Huisken, J., Ferrer, T., Arnaout, R., Scott, I., Beis, D., Xiao, T., Baier, H. et al. (2008). Genetic and physiologic dissection of the vertebrate cardiac conduction system. PLoS Biol. 6, e109.

Chin, A. J., Saint-Jeannet, J.-P. and Lo, C. W. (2012). How insights from cardiovascular developmental biology have impacted the care of infants and children with congenital heart disease. Mech. Dev. 129, 75-97.

Corada, M., Morini, M. F. and Dejana, E. (2014). Signaling pathways in the specification of arteries and veins. Arterioscler. Thromb. Vasc. Biol. 34 2372-2377

Culver, J. C. and Dickinson, M. E. (2010). The effects of hemodynamic force on embryonic development. Microcirculation 17, 164-178

de la Pompa, J. L. and Epstein, J. A. (2012). Coordinating tissue interactions: Notch signaling in cardiac development and disease. Dev. Cell 22, 244-254

Delling, M., DeCaen, P. G., Doerner, J. F., Febvay, S. and Clapham, D. E. (2013) Primary cilia are specialized calcium signalling organelles. Nature 504, 311-314.

Dietrich, A.-C., Lombardo, V. A. Veerkamp, J., Priller, F. and AbdelilahSeyfried, S. (2014). Blood flow and Bmp signaling control endocardial chamber morphogenesis. Dev. Cell 30, 367-377.

Egorova, A. D., van der Heiden, K., Poelmann, R. E. and Hierck, B. P. (2012) Primary cilia as biomechanical sensors in regulating endothelial function. Differentiation 83, S56-S61.

Firestone, A. J., Weinger, J. S., Maldonado, M., Barlan, K., Langston, L. D., O'Donnell, M., Gelfand, V. I., Kapoor, T. M. and Chen, J. K. (2012). Smallmolecule inhibitors of the AAA+ ATPase motor cytoplasmic dynein. Nature 484 125-129.

Fishman, M. C. and Chien, K. R. (1997). Fashioning the vertebrate heart: earliest embryonic decisions. Development 124, 2099-2117.

Gassmann, M., Casagranda, F., Orioli, D., Simon, H., Lai, C., Klein, R. and Lemke, G. (1995). Aberrant neural and cardiac development in mice lacking the ErbB4 neuregulin receptor. Nature 378, 390-394.

Gerdes, J. M., Davis, E. E. and Katsanis, N. (2009). The vertebrate primary cilium in development, homeostasis, and disease. Cell 137, 32-45.

Goetz, J. G., Steed, E., Ferreira, R. R., Roth, S., Ramspacher, C., Boselli, F., Charvin, G., Liebling, M., Wyart, C., Schwab, Y. et al. (2014). Endothelial cilia mediate low flow sensing during zebrafish vascular development. Cell Rep. 6 799-808

Grego-Bessa, J., Luna-Zurita, L., del Monte, G., Bolós, V., Melgar, P., Arandilla, A., Garratt, A. N., Zang, H., Mukouyama, Y.-S., Chen, H. et al. (2007). Notch signaling is essential for ventricular chamber development. Dev. Cell 12, 415-429.

Gridley, T. (2010). Notch signaling in the vasculature. Curr. Top. Dev. Biol. 92 277-309.

Hahn, C. and Schwartz, M. A. (2009). Mechanotransduction in vascular physiology and atherogenesis. Nat. Rev. Mol. Cell Biol. 10, 53-62.

Harvey, R. P. (2002). Organogenesis: Patterning the vertebrate heart. Nat. Rev. Genet. 3, 544-556

Heckel, E., Boselli, F., Roth, S., Krudewig, A., Belting, H.-G., Charvin, G. and Vermot, J. (2015). Oscillatory flow modulates mechanosensitive klf2a expression through trpv4 and trpp2 during heart valve development. Curr. Biol. 25, 1354-1361.

Hierck, B. P., Van der Heiden, K., Alkemade, F. E., Van de Pas, S., Van Thienen, J. V., Groenendijk, B. C. W., Bax, W. H., Van der Laarse, A., DeRuiter, M. C. Horrevoets, A. J. G. et al. (2008). Primary cilia sensitize endothelial cells for fluid shear stress. Dev. Dyn. 237, 725-735

Hove, J. R., Köster, R. W., Forouhar, A. S., Acevedo-Bolton, G., Fraser, S. E. and Gharib, M. (2003). Intracardiac fluid forces are an essential epigenetic factor for embryonic cardiogenesis. Nature 421, 172-177.

Huang, C.-J., Tu, C.-T., Hsiao, C.-D., Hsieh, F.-J. and Tsai, H.-J. (2003). Germ-line transmission of a myocardium-specific GFP transgene reveals critical regulatory elements in the cardiac myosin light chain 2 promoter of zebrafish. Dev. Dyn. 228 $30-40$

Iomini, C., Tejada, K., Mo, W., Vaananen, H. and Piperno, G. (2004). Primary cilia of human endothelial cells disassemble under laminar shear stress. J. Cell Biol. 164, 811-817.

Itoh, M., Kim, C.-H., Palardy, G., Oda, T., Jiang, Y.-J., Maust, D., Yeo, S.-Y., Lorick, K., Wright, G. J., Ariza-McNaughton, L. et al. (2003). Mind bomb is a ubiquitin ligase that is essential for efficient activation of Notch signaling by Delta. Dev. Cell 4, 67-82.

Jenni, R., Rojas, J. and Oechslin, E. (1999). Isolated noncompaction of the myocardium. N. Engl. J. Med. 340, 966-967.

Jiang, Y. J., Brand, M., Heisenberg, C. P., Beuchle, D., Furutani-Seiki, M., Kelsh R. N., Warga, R. M., Granato, M., Haffter, P., Hammerschmidt, M. et al. (1996) Mutations affecting neurogenesis and brain morphology in the zebrafish, Danio rerio. Development 123, 205-216.

Jin, S.-W., Beis, D., Mitchell, T., Chen, J.-N. and Stainier, D. Y. R. (2005). Cellular and molecular analyses of vascular tube and lumen formation in zebrafish Development 132, 5199-5209.

Kalogirou, S., Malissovas, N., Moro, E., Argenton, F., Stainier, D. Y. R. and Beis, D. (2014). Intracardiac flow dynamics regulate atrioventricular valve morphogenesis. Cardiovasc. Res. 104, 49-60.

Kattman, S. J., Huber, T. L. and Keller, G. M. (2006). Multipotent flk-1+ cardiovascular progenitor cells give rise to the cardiomyocyte, endothelial, and vascular smooth muscle lineages. Dev. Cell 11, 723-732

Kramer-Zucker, A. G., Olale, F., Haycraft, C. J., Yoder, B. K., Schier, A. F. and Drummond, I. A. (2005). Cilia-driven fluid flow in the zebrafish pronephros, brain and Kupffer's vesicle is required for normal organogenesis. Development 132 1907-1921.

Lai, D., Liu, X., Forrai, A., Wolstein, O., Michalicek, J., Ahmed, I., Garratt, A. N. Birchmeier, C., Zhou, M., Hartley, L. et al. (2010). Neuregulin 1 sustains the gene regulatory network in both trabecular and nontrabecular myocardium. Circ. Res. 107, 715-727.

Lee, K.-F., Simon, H., Chen, H., Bates, B., Hung, M.-C. and Hauser, C. (1995). Requirement for neuregulin receptor erbB2 in neural and cardiac development. Nature 378, 394-398.

Lee, J., Moghadam, M. E., Kung, E., Cao, H., Beebe, T., Miller, Y., Roman, B. L., Lien, C.-L., Chi, N. C., Marsden, A. L. et al. (2013). Moving domain computational fluid dynamics to interface with an embryonic model of cardiac morphogenesis. PLOS ONE 8, e72924.

Lin, Y.-F., Swinburne, I. and Yelon, D. (2012). Multiple influences of blood flow on cardiomyocyte hypertrophy in the embryonic zebrafish heart. Dev. Biol. 362 242-253.

Liu, J. and Stainier, D. Y. R. (2010). Tbx5 and Bmp signaling are essential for proepicardium specification in zebrafish. Circ. Res. 106, 1818-1828.

Liu, J., Bressan, M., Hassel, D., Huisken, J., Staudt, D., Kikuchi, K., Poss, K. D. Mikawa, T. and Stainier, D. Y. R. (2010). A dual role for ErbB2 signaling in cardiac trabeculation. Development 137, 3867-3875.

Lunt, S. C., Haynes, T. and Perkins, B. D. (2009). Zebrafish ift57, ift88, and ift172 intraflagellar transport mutants disrupt cilia but do not affect hedgehog signaling. Dev. Dyn. 238, 1744-1759.

Meyer, D. and Birchmeier, C. (1995). Multiple essential functions of neuregulin in development. Nature 378, 386-390.

Milan, D. J., Giokas, A. C., Serluca, F. C., Peterson, R. T. and MacRae, C. A (2006). Notch1b and neuregulin are required for specification of central cardiac conduction tissue. Development 133, 1125-1132.

Milgrom-Hoffman, M., Harrelson, Z., Ferrara, N., Zelzer, E., Evans, S. M. and Tzahor, E. (2011). The heart endocardium is derived from vascular endothelial progenitors. Development 138, 4777-4787.

Moorman, A. F. and Christoffels, V. M. (2003). Cardiac chamber formation: development, genes, and evolution. Physiol. Rev. 83, 1223-1267.

Moretti, A., Caron, L., Nakano, A., Lam, J. T., Bernshausen, A., Chen, Y., Qyang, Y., Bu, L., Sasaki, M., Martin-Puig, S. et al. (2006). Multipotent embryonic isl1+ progenitor cells lead to cardiac, smooth muscle, and endothelial cell diversification. Cell 127, 1151-1165.

Neugebauer, J. M. and Yost, H. J. (2014). FGF signaling is required for brain leftright asymmetry and brain midline formation. Dev. Biol. 386, 123-134.

Ninov, N., Borius, M. and Stainier, D. Y. R. (2012). Different levels of Notch signaling regulate quiescence, renewal and differentiation in pancreatic endocrine progenitors. Development 139, 1557-1567.

Olson, E. N. and Srivastava, D. (1996). Molecular pathways controlling heart development. Science 272, 671-676.

Parsons, M. J., Pisharath, H., Yusuff, S., Moore, J. C., Siekmann, A. F., Lawson N. and Leach, S. D. (2009). Notch-responsive cells initiate the secondary transition in larval zebrafish pancreas. Mech. Dev. 126, 898-912.

Peralta, M., Steed, E., Harlepp, S., González-Rosa, J. M., Monduc, F., ArizaCosano, A., Cortés, A., Rayón, T., Gómez-Skarmeta, J. L., Zapata, A. et al. (2013). Heartbeat-driven pericardiac fluid forces contribute to epicardium morphogenesis. Curr. Biol. 23, 1726-1735.

Peshkovsky, C., Totong, R. and Yelon, D. (2011). Dependence of cardiac trabeculation on neuregulin signaling and blood flow in zebrafish. Dev. Dyn. 240 446-456.

Rothschild, S. C., Easley, C. A., Francescatto, L., Lister, J. A., Garrity, D. M. and Tombes, R. M. (2009). Tbx5-mediated expression of $\mathrm{Ca}(2+) /$ calmodulindependent protein kinase II is necessary for zebrafish cardiac and pectoral fin morphogenesis. Dev. Biol. 330, 175-184.

Roy, S. (2012). Cilia and Hedgehog: when and how was their marriage solemnized? Differentiation 83, S43-S48. 
Samsa, L. A., Yang, B. and Liu, J. (2013). Embryonic cardiac chamber maturation trabeculation, conduction, and cardiomyocyte proliferation. Am. J. Med. Genet. C Semin. Med. Genet. 163, 157-168.

Sasai, N. and Briscoe, J. (2012). Primary cilia and graded Sonic Hedgehog signaling. Wiley Interdiscip. Rev. Dev. Biol. 1, 753-772.

Scheer, N. and Campos-Ortega, J. A. (1999). Use of the Gal4-UAS technique for targeted gene expression in the zebrafish. Mech. Dev. 80, 153-158.

Scheer, N., Groth, A., Hans, S. and Campos-Ortega, J. A. (2001). An instructive function for Notch in promoting gliogenesis in the zebrafish retina. Development 128, 1099-1107.

Schneider, C. A., Rasband, W. S. and Eliceiri, K. W. (2012). NIH Image to ImageJ: 25 years of image analysis. Nat. Methods 9, 671-675.

Sedmera, D., Pexieder, T., Rychterova, V., Hu, N. and Clark, E. B. (1999). Remodeling of chick embryonic ventricular myoarchitecture under experimentally changed loading conditions. Anat. Rec. 254, 238-252.

Sedmera, D., Pexieder, T., Vuillemin, M., Thompson, R. P. and Anderson, R. H (2000). Developmental patterning of the myocardium. Anat. Rec. 258, 319-337.

Sehnert, A. J., Huq, A., Weinstein, B. M., Walker, C., Fishman, M. and Stainier, D. Y. R. (2002). Cardiac troponin T is essential in sarcomere assembly and cardiac contractility. Nat. Genet. 31, 106-110.

Slough, J., Cooney, L. and Brueckner, M. (2008). Monocilia in the embryonic mouse heart suggest a direct role for cilia in cardiac morphogenesis. Dev. Dyn 237, 2304-2314.

Sorescu, G. P., Sykes, M., Weiss, D., Platt, M. O., Saha, A., Hwang, J., Boyd, N. Boo, Y. C., Vega, J. D., Taylor, W. R. et al. (2003). Bone morphogenic protein 4 produced in endothelial cells by oscillatory shear stress stimulates an inflammatory response. J. Biol. Chem. 278, 31128-31135.

Stainier, D. Y., Weinstein, B. M., Detrich, H. W., III, Zon, L. I. and Fishman, M. C. (1995). Cloche, an early acting zebrafish gene, is required by both the endothelia and hematopoietic lineages. Development 121, 3141-3150.

Stainier, D. Y. R., Beis, D., Jungblut, B. and Bartman, T. (2002). Endocardial cushion formation in zebrafish. Cold Spring Harb. Symp. Quant. Biol. 67, 49-56.

Stanton, B. Z. and Peng, L. F. (2010). Small-molecule modulators of the Sonic Hedgehog signaling pathway. Mol. Biosyst. 6, 44-54.

Staudt, D. and Stainier, D. (2012). Uncovering the molecular and cellular mechanisms of heart development using the zebrafish. Annu. Rev. Genet. 46 397-418.

Staudt, D. W., Liu, J., Thorn, K. S., Stuurman, N., Liebling, M. and Stainier, D. Y. R. (2014). High-resolution imaging of cardiomyocyte behavior reveals two distinct steps in ventricular trabeculation. Development 141, 585-593.

Sweet, D. T., Chen, Z., Wiley, D. M., Bautch, V. L. and Tzima, E. (2012). The adaptor protein Shc integrates growth factor and ECM signaling during postnatal angiogenesis. Blood 119, 1946-1955
Sweet, D. T., Chen, Z., Givens, C. S., Owens, A. P., III, Rojas, M. and Tzima, E. (2013). Endothelial Shc regulates arteriogenesis through dual control of arteria specification and inflammation via the notch and nuclear factor-kappa-light-chainenhancer of activated B-cell pathways. Circ. Res. 113, 32-39.

Tian, Y. and Morrisey, E. E. (2012). Importance of myocyte-nonmyocyte interactions in cardiac development and disease. Circ. Res. 110, 1023-1034.

Tsujikawa, M. and Malicki, J. (2004). Intraflagellar transport genes are essential for differentiation and survival of vertebrate sensory neurons. Neuron 42, 703-716.

Van der Heiden, K., Groenendijk, B. C. W., Hierck, B. P., Hogers, B., Koerten, H. K., Mommaas, A. M., Gittenberger-de Groot, A. C. and Poelmann, R. E. (2006). Monocilia on chicken embryonic endocardium in low shear stress areas. Dev. Dyn. 235, 19-28.

Van der Heiden, K., Egorova, A. D., Poelmann, R. E., Wentzel, J. J. and Hierck B. P. (2011). Role for primary cilia as flow detectors in the cardiovascular system. Int. Rev. Cell Mol. Biol. 290, 87-119.

van Eeden, F. J., Granato, M., Schach, U., Brand, M., Furutani-Seiki, M., Haffter, P., Hammerschmidt, M., Heisenberg, C. P., Jiang, Y. J., Kane, D. A. et al (1996). Mutations affecting somite formation and patterning in the zebrafish, Danio rerio. Development 123, 153-164.

Vermot, J., Forouhar, A. S., Liebling, M., Wu, D., Plummer, D., Gharib, M. and Fraser, S. E. (2009). Reversing blood flows act through klf2a to ensure norma valvulogenesis in the developing heart. PLOS Biol. 7, e1000246.

Wagner, M. and Siddiqui, M. A. (2007). Signal transduction in early heart development (II): ventricular chamber specification, trabeculation, and heart valve formation. Bull. Exp. Biol. Med. 232, 866-880.

Wang, Y., Nakayama, M., Pitulescu, M. E., Schmidt, T. S., Bochenek, M. L., Sakakibara, A., Adams, S., Davy, A., Deutsch, U., Lüthi, U. et al. (2010). Ephrin-B2 controls VEGF-induced angiogenesis and lymphangiogenesis. Nature 465, 483-486.

Westerfield, M. (2000). The Zebrafish Book: a Guide for the Laboratory Use of Zebrafish (Danio rerio). Eugene, OR: University of Oregon Press.

Wilson, C. W. and Stainier, D. Y. R. (2010). Vertebrate Hedgehog signaling: cilia rule. BMC Biol. 8, 102.

Yang, J., Hartjes, K. A., Nelson, T. J. and Xu, X. (2014). Cessation of contraction induces cardiomyocyte remodeling during zebrafish cardiogenesis Am. J. Physiol. Heart Circ. Physiol. 306, H382-H395.

Yelon, D. (2001). Cardiac patterning and morphogenesis in zebrafish. Dev. Dyn. 222, 552-563.

Yoshiba, S., Shiratori, H., Kuo, I. Y., Kawasumi, A., Shinohara, K., Nonaka, S. Asai, Y., Sasaki, G., Belo, J. A., Sasaki, H. et al. (2012). Cilia the node of mouse embryos sense fluid flow for left-right determination via Pkd2. Science 338 226-231 